\title{
Prevalence, Awareness and Control of Hypertension in a Well-Educated Professional Group in Nepal
}

\author{
Mani Prasad Gautam, ${ }^{1}$ Usha Ghimire,${ }^{2}$ Krishna Maya Shrestha, ${ }^{1}$ Bijaya Paudel, ${ }^{1}$ Prakash Khatiwada, ${ }^{1}$ \\ Bhojraj Adhikari ${ }^{1}$
}

${ }^{1}$ Department of Medicine, Bharatpur Hospital, Chitawan, Nepal
${ }^{2}$ Communication and Management Institute Private Limited, Kathmandu, Nepal

Corresponding Author: Mani Prasad Gautam

Department of Medicine,

Bharatpur Hospital, Chitawan, Nepal,

Email Address: manigautam@gmail.com

\begin{abstract}
Background and Aims: Hypertension continues to be the major modifiable risk factor leading to premature death due to cardiovascular diseases. Therefore awareness, timely diagnosis, treatment and control of hypertension in primary care are crucial for reducing disease burden. In this respect, authentic data on these issues are paramount of importance. The aim of this study was to address this gap in a well-educated group of Nepalese subjects

Methods: : A cross-sectional study in the delegates of the national level meeting of a professional group - lawyers was conducted in 2015 February. Standard criteria were used to define hypertension and blood pressure goal. Awareness and treatment were based on self-report.

Results: A total of 249 subjects were included in the study. The prevalence of hypertension was $18.88 \%$. Among hypertensive, $70 \%$ subjects were newly detected and they were unaware of their blood pressure status before. Remaining $30 \%$ were known cases of hypertension and among them only $64 \%$ were on medicine and $36 \%$ were not despite awareness of hypertension. Only $55.5 \%$ of them on medicine had achieved blood pressure goal.

Conclusion: The scenario of hypertension in Nepal even in educated group is very alarming with majority of them being unaware of the hypertension status, more than one third not taking medicine and nearly half on medicine not achieving desired blood pressure goal. From this, the situation obviously seems more warning in general population and needs well-designed assessment.
\end{abstract}

Keywords : Awareness; Hypertension; Management; Prevalence.

\section{Introduction}

Hypertension is the major modifiable risk factor leading to premature death due to cardio and cerebrovascular diseases. ${ }^{1}$ It was estimated that nearly 1 billion people were living with hypertension in 2000 and the disease burden is predicted to affect 1.56 billion by $2025 .^{2}$ The peculiarity with hypertension is that the mortality and morbidity start to rise in linear pattern starting from $115 / 75 \mathrm{~mm} \mathrm{Hg},{ }^{3}$ so that there is no threshold of safe blood pressure. Small increment in blood pressure above $115 / 75$ has been shown to substantial changes in cardio and cerebrovascular disease burden. ${ }^{4}$ The pharmacological interventions along with lifestyle and behavioral changes can effectively reduce the cardiovascular events attributed to hypertension. ${ }^{5}$ Therefore timely diagnosis, treatment and control of hypertension in primary care are crucial for reducing disease burden as hypertension has been found to be a strong coronary risk factor. ${ }^{6}$ However, majority with hypertension, despite the available evidences, are not effectively treated and controlled to the recommended blood pressure goals. ${ }^{7}$

Most of the published studies from Nepal are focused on prevalence; studies on awareness, treatment and control rate are lacking. The initial data of prevalence ranging from $5.3 \%$ to $9.9 \%$ in the different regions of the country were based on community prevalence study ${ }^{8}$ and level as defined ${ }^{9}$ by World Health Organization at that time. ${ }^{10,11}$ In contrast to this, more recent studies showed almost three times increase in prevalence. ${ }^{12,13}$ Regarding awareness and treatment, a study ${ }^{14}$ recorded the awareness, treatment, and control rates of $41.1 \%, 26 \%$, and $6 \%$, respectively with the overall prevalence of $19.7 \%$. In a large international study involving 152996 adults from all over the World, ${ }^{15}$ low education was associated with lower rates of awareness, treatment, and control only in low-income countries (LICs) with prevalence of hypertension $52 \%$, awareness $40.8 \%, 31.7 \%$ subjects on treatment and $12.7 \%$ had controlled blood pressure level.

As Nepal is a representative of LICs, we assume that the picture with prevalence, awareness, treatment and control in an educated group definitely will be better than on population level. Therefore, this study was designed to evaluate these issues in a well-educated group of Nepalese subjects.

\section{Method}

A cross-sectional study was designed. The subjects were the volunteers among the participants of the national level meeting 
of lawyers held in Ratnanagar, Chitawan, Nepal in 2015 February. A standard proforma containing demographic and clinical details was used. Verbal consent for the use of clinical and demographic data was taken. Blood pressure was measured by the conventional auscultatory method with an aneroid sphygmomanometer with standard sized cuff $(12 \times 34 \mathrm{~cm})$. The blood pressure measurement was taken in the seated position, quietly in a chair with feet on the floor and an arm support at the level of heart. Qualified, trained and experienced paramedics took Blood Pressure measurements. The definition of hypertension was adapted from the guidelines of the Seventh Report of the Joint National Committee on Prevention, Detection, Evaluation and Treatment of High Blood Pressure, ${ }^{5}$ that is, systolic blood pressure (SBP) $\geq 140 \mathrm{~mm} \mathrm{Hg}$ or diastolic blood pressure (DBP) $\geq 90 \mathrm{~mm} \mathrm{Hg}$ and/or concomitant use of antihypertensive medications. Subjects having high blood pressure in first reading were subjected for second reading and higher one was selected. As it was a snap shot type of blood pressure measurement in an outreach setting, single threshold reading either in systolic $(140 \mathrm{mmHg})$ or diastolic $(90 \mathrm{mmHg})$ was taken as normal, if both were in threshold level, subject was considered as hypertensive. Any reading above the threshold level either systolic or diastolic or both were considered hypertensive. Awareness was based on the self-report of known status regarding blood pressure level independent of the treatment and adherence, no medical documents were asked for. Treatment was also based on self-reports; neither independent verification nor medical document was sought. Subjects having incomplete data were excluded from the study. Similarly non-delegates were also excluded. Simple statistical calculations using MS Excel 2010 were done and the studied parameters; prevalence, awareness, treatment and control, were expressed in percentages. No comparisons with other data were conducted. No interobserver and intraobserver variability were studied.

\section{Results}

A total of 287 subjects were screened. As the 32 subjects had incomplete data in the filled proforma and six subjects were nondelegates, they were excluded from the study. Remaining 249 subjects were included in the study (Figure 1).

Mean age was $44.06 \pm 7.48$ years and ranged from 26 to 66 years with male to female ratio of $11.45: 1$. The prevalence of hypertension was $18.88 \%$. The mean age in hypertensives was 47.45 (7.47) years with male to female ratio of 22.5:1. Mean SBP, DBP, MAP (mean arterial pressure) were 142.98 (11.02), 94 (6.37) and 110.37 (7.92) $\mathrm{mmHg}$ respectively. In nonhypertensive subjects the mean age, male to female ratio, SBP, DBP and MAP were 43.28 (7.28) years, 10.2:1, 120.02 (10.32), 79.43 (7.72) and 92.96 (8.59) $\mathrm{mmHg}$ respectively. Among hypertensives, 70\% subjects were unaware of their blood pressure status before. Remaining $30 \%$ were known cases of hypertension, all male and only $64 \%$ were on medicine and $36 \%$ were not despite known hypertension status. Only $55.5 \%$ of them on medicine had achieved blood pressure goal. Various parameters of the different category subjects are given in table 1 and 2 and these differences are obvious if we take their status in consideration. Although the female participants were less represented in the study, but not a single hypertensive female was aware of her blood pressure status. In overall, the prevalence of hypertension was $18.88 \%$. Only $29.79 \%$ subjects were aware of their blood pressure status, $19.15 \%$ subjects were on treatment and only $10.64 \%$ subjects were controlled.

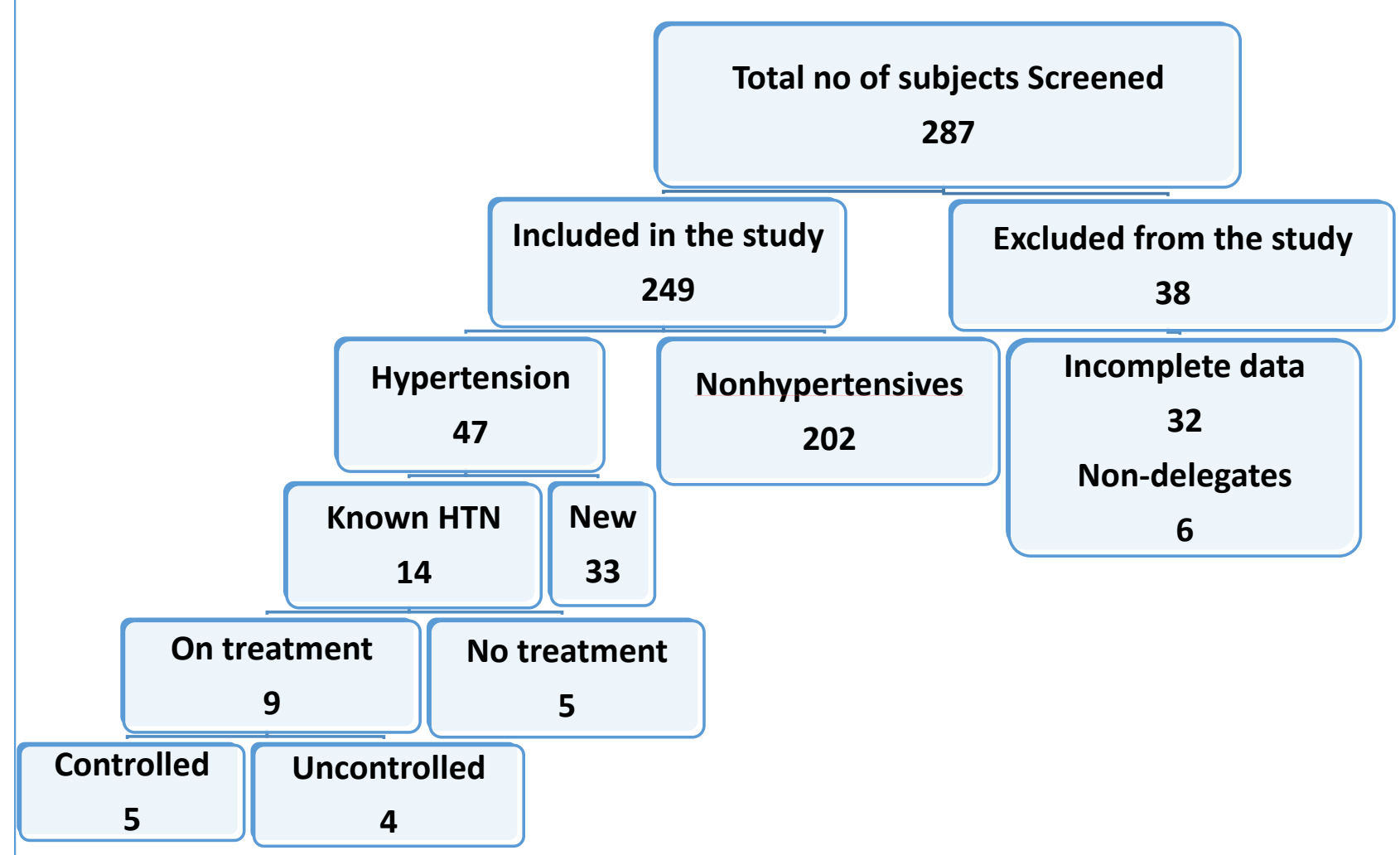

Figure 1. Overview of Study Subjects 


\begin{tabular}{|c|c|c|c|c|c|}
\hline & Total Subjects & HTN & Non HTN & Known HTN & New HTN \\
\hline $\begin{array}{l}\text { Number of } \\
\text { Subjects }\end{array}$ & 249 & 47 & 202 & 14 & 33 \\
\hline Age in Yrs & $44.06(7.48)$ & $47.45(7.47)$ & $43.28(7.28)$ & $48.08(7.98)$ & $47.19(7.36)$ \\
\hline $\mathrm{M}: \mathrm{F}$ & $11.45: 1$ & $22.5: 1$ & $10.2: 1$ & All Male & $16.5: 1$ \\
\hline SBP mmHg & $124.35(13.78)$ & $142.98(11.02)$ & $120.02(10.32)$ & $142.86(16.84)$ & $143.03(7.7)$ \\
\hline DBP mmHg & $82.18(9.41)$ & $94(6.37)$ & $79.43(7.72)$ & $94.57(9.23)$ & $93.76(4.84)$ \\
\hline MAP mmHg & $96.24(10.87)$ & $110.37(7.92)$ & $92.96(8.59)$ & $110.67(11.77)$ & $110.18(5.79)$ \\
\hline
\end{tabular}

\begin{tabular}{|c|c|c|c|c|}
\hline Parameters & Known HTN on T/T & $\begin{array}{c}\text { Known HTN not on } \\
\text { T/T }\end{array}$ & On T/T Controlled & On T/T not Controlled \\
\hline Number of Subjects & 9 & 5 & 5 & 4 \\
\hline Age Years & $48.25(9.72)$ & $47.8(5.07)$ & $51.75(13.33)$ & $44.75(3.2)$ \\
\hline M:F & All Male & All Male & All Male & All Male \\
\hline SBP mmHg & $135.56(11.3)$ & $156(18.17)$ & $128(4.47)$ & $145(10.0)$ \\
\hline DBP mmHg & $89.78(6.74)$ & $103.2(6.42)$ & $84(5.48)$ & $94.5(5.26)$ \\
\hline MAP mmHg & $105.04(8.26)$ & $120.8(10.34)$ & $98.67(5.14)$ & $111.33(6.84)$ \\
\hline
\end{tabular}

\section{Discussion}

This study revealed the actual scenario of hypertension in a welleducated group of subjects. If the findings of the study by Chow et $\mathrm{a}^{15}$, which had shown the low education was associated with lower rates of awareness, treatment, and control in LICs, is taken into consideration, the actual scenario in general population seems alarming. Even on the well-educated group the overall prevalence of $18.88 \%$, awareness of $29.79 \%$, treatment of only $19.15 \%$ and control of blood pressure in $10.64 \%$ of hypertensive subjects as shown in current study is unique from various angles.

The prevalence of hypertension in current study is comparatively in between if it is compared with other community based studies. The initial studies had shown lower prevalence of hypertension (5.3-9.9\%). ${ }^{9}$ But the criteria used in these studies, WHO criteria practiced at that time where hypertension was defined as the blood pressure level $\geq 160 / 95 \mathrm{mmHg}$, was higher than the current definition of $\geq 140 / 90 \mathrm{mmHg}$ and lower prevalence is justifiable to some extent. More recent community based studies showed higher prevalence of hypertension, defined as per JNC VII criteria; three times higher than previously recorded in suburban Kathmandu ${ }^{12}$ and 34\% in Eastern Nepal. ${ }^{13}$

A house-to-house survey in a suburban area of Kathmandu valley from February to June 2005 in adult population (age $\geq 18$ years) showed the overall prevalence of hypertension $19.7 \%$ $(22.2 \%$ in men and $17.3 \%$ in women, $\mathrm{p}<0.05)$. Prevalence of hypertension in age group of $\geq 40$ years was $36 \%$. Awareness, treatment, and control rates were $41.1 \%, 26 \%$, and $6 \%$ respectively. ${ }^{14}$ In present study, the awareness and treatment rate even in educated group was less; $29.79 \%$ and $19.15 \%$ respectively. However the control rate was $10.64 \%$, which is slightly better than in community based study. The situation is far better in developed countries. In a study among residents accessing community health services in Beijing, the prevalence of hypertension was $53.5 \%$. The rates of awareness, treatment and control of hypertension were $70.0 \%, 62.1 \%$ and $29.6 \%$, respectively. ${ }^{16}$ Similar figures were also reported for USA, Canada and England. ${ }^{17}$ The prevalence of hypertension in USA, Canada and England was 29\%, 19.5\% and 30\% respectively.The highest awareness rate $(83 \%)$ was in Canada followed by USA (81\%) and then by England (65\%). The treatment rate was also higher in Canada (80\%) followed by USA (74\%) and England $(51 \%)$. Similar pattern of control rate was evident in these countries (66\%, 53\%, and 27\% in Canada, USA and England respectively). However the situation in other developing countries is comparable to our current findings. In India, in a communitybased study in Kerala by Thankappan et al, the overall prevalence of hypertension was $36.7 \%$ (95\% CI: $35.3-38.0$; men: $36.0 \%$ and women $37.2 \%$ ). Among hypertensives, $24 \%$ were aware of the condition, $20 \%$ were on treatment, and $6.4 \%$ achieved effective blood pressure control. ${ }^{18}$ These findings are largely consistent with our recent observation. The scenario in elderly group might be different which should be studied separately. In a study on elderly population conducted in Bangladesh the overall prevalence of hypertension was $65 \%$. Among study subjects who had hypertension, $45 \%$ were aware of their condition, $40 \%$ were taking anti-hypertensive medications, but only $10 \%$ achieved the desired goal. ${ }^{19}$ Our study was unable to reveal the scenario in elderly people, as our participants were younger.

One of the largest study on prevalence, awareness, treatment and control of hypertension, the PURE (Prospective Urban Rural Epidemiology) Study, ${ }^{15}$ a cross-sectional study of 153,996 adults (complete data for this analysis on 142,042) aged 35 to 70 years, recruited between January 2003 and December 2009 from 628 communities in 3 high-income countries (HIC), 10 upper-middleincome and low-middle-income countries (UMIC and LMIC), and 4 low-income countries (LIC) has also shown similar to our study findings. In overall, among the 142,042 participants, 57,840 $(40.8 \%)$ had hypertension and $26,877(46.5 \%)$ were aware of the diagnosis. Of those who were aware of the diagnosis, the majority $(23,510[87.5 \%])$ were receiving pharmacological treatments, but only a minority of those receiving treatment were controlled $(7,634$ [32.5\%]). The percentages aware (49.0\% in HICs, $52.5 \%$ in UMICs, $43.6 \%$ in LMICs, and $40.8 \%$ in LICs) and treated (46.7\% in HICs, $48.3 \%$ in UMICs, $36.9 \%$ in LMICs, and $31.7 \%$ in LICs) were lower in LICs compared with all other countries for awareness $(\mathrm{P}<0.001)$ and treatment $(\mathrm{P}<0.001)$. Awareness, treatment, and control of 
hypertension were higher in urban communities compared with rural ones in LICs (urban vs rural, $\mathrm{P}<0.001$ ) and LMICs (urban vs rural, $\mathrm{P}<0.001$ ), but similar for other countries. Nepal, a representative of LICs, the prevalence, awareness, treatment, overall control, and control rate among treated of $52 \%, 40.8 \%, 31.7 \% 12.7 \%$ and $40.2 \%$ respectively for LICs as reported in PURE study was in contrast to the findings of present study $(18.88 \%, 29.79 \%, 19.15 \%, 10.64 \%$ and $55.5 \%$ respectively) with significantly lower prevalence, awareness and treatment rate. The overall control rate and control rate among treated were almost similar. In the PURE study, low education was associated with lower rates of awareness, treatment, and control in LICs, but not in other countries. ${ }^{15}$ So, it seems the real scenario in community is worse than the current findings in educated group of people. The lower prevalence, awareness and treatment in current study could be because of younger participants in our study.

This is a small study and it has some limitations. This is a cross-sectional study and is based on the single day measurement of blood pressure. Participants were not chosen randomly and all who came voluntarily for the blood pressure measurement were included, that might have impact on higher prevalence, awareness and better management indicators. Although measured twice in those having high blood pressure in first reading, those aware of hypertensive status and subjects taking treatment, the impact of recently taken diet or smoking and other confounding variables were not taken into consideration. The impact of methodology based on choosing higher reading of two recordings should also be considered as it may have caused higher prevalence of hypertension in this cohort. The awareness and treatment was based on self-report and chance of recall bias and other relevant biases should be considered while interpreting the current findings. Limitations inherent to the camp based rather than community based design and sample size should also be taken into consideration while extrapolating these data.

\section{Conclusion}

The scenario of hypertension in Nepal even in educated group is very alarming with a prevalence of $18.88 \%$. Among them, $70 \%$ subjects are unaware of the hypertension status. Moreover, despite awareness, $36 \%$ subjects are not taking any medicine and only $55.5 \%$ subjects on medicine has achieved target blood pressure goal. This study calls for a larger community based prevalence and awareness study.

\section{References}

1. WHO. World Health Organization, World Health Statistics 2012. Geneva: World Health Organization, 2012.

2. Kearney PM, Whelton M, Reynolds K et al. Global burden of hypertension: analysis of worldwide data. Lancet 2005;365:217-23.

3. Lewington S, Clarke R, Qizilbash N, et al. Age-specific relevance of usual blood pressure to vascular mortality: a meta-analysis of individual data for one million adults in 61 prospective studies. Lancet 2002;360:1903-13.

4. Law MR, Morris JK, Wald NJ. Use of blood pressure lowering drugs in the prevention of cardiovascular disease: meta-analysis of 147 randomized trials in the context of expectations from prospective epidemiological studies. BMJ 2009;338:b1665.

5. Chobanian AV, Bakris GL, Black HR, et al. National Heart, Lung, and Blood Institute Joint National Committee on Prevention, Detection, Evaluation, and Treatment of High
Blood Pressure; National High Blood Pressure Education Program Coordinating Committee. The Seventh Report of the Joint National Committee on Prevention, Detection, Evaluation, and Treatment of High Blood Pressure: the JNC 7 report. JAMA2003;289:2560-72.

6. Gautam MP, Gautam S, Ghimire U, et al. Endothelial dysfunction and the role of hypertension in Nepalese subjects with major coronary risk factors. Nepalese Heart Journal 2016; 13(1): 13-8.

7. Kearney PM, Whelton M, Reynolds K, et al. Worldwide prevalence of hypertension: a systematic review.J Hypertens 2004:22:11-9.

8. Pandey MR. Hypertension in Nepal. Biblthea Cardio. 1987;42:68-76.

9. World Health Organization. Arterial Hypertension. Report of a WHO Expert Committee. WHO Technical Report Series 628. World Health Organization; Geneva, Switzerland: 1978.

10. Pandey MR, Dhungel S. Prevalence of hypertension in an urban community of Nepal. Journal of Nepal Medical Association. 1983;21:1-5.

11. Pandey MR, Upadhyay LR, Dhungel S. Prevalence of hypertension in a rural community in Nepal. Indian Heart J. 1981;33:284-9.

12. Vaidya A, Pathak RP, Pandey MR. Prevalence of hypertension in Nepalese community triples in 25 years: a repeat cross-sectional study in rural Kathmandu. Indian Heart J. 2012; 64(2): 128-31.

13. Sharma SK, Ghimire A, Radhakrishnan J, et al. Prevalence of hypertension, obesity, diabetes, and metabolic syndrome in Nepal. International Journal of Hypertension 2011; 2011: 821971. doi:10.4061/2011/821971.

14. Sharma D, KC MB, Rajbhandari S, et al. Study of Prevalence, Awareness, and control of Hypertension in a Suburban Area of Kathmandu, Nepal. Indian Heart J. 2006;58(1):34-7.

15. Chow CK, Teo KK, Rangarajan S, et al for the PURE (Prospective Urban Rural Epidemiology) Study investigators. Prevalence, Awareness, Treatment, and Control of Hypertension in Rural and Urban Communities in High-, Middle-, and Low-Income Countries. JAMA. 2013;310(9):959-68.

16. Jiang B, Liu H, Ru X, et al. Hypertension detection, management, control and associated factors among residents accessing community health services in Beijing. Sci Rep. 2014;4:4845. doi: 10.1038/srep04845.

17. Joffres M, Falaschetti E, Gillespie C et al. Hypertension prevalence, awareness, treatment and control in national surveys from England, the USA and Canada, and correlation with stroke and ischaemic heart disease mortality: a crosssectional study. BMJOpen 2013;3:e003423 doi:10.1136/ bmjopen-2013-003423.

18. Thankappan KR, Sivasankaran S, Khader SA, et al. Prevalence, Correlates, Awareness, Treatment, and Control of Hypertension in Kumarakom, Kerala: Baseline Results of a Community-Based Intervention Program. Indian Heart J 2006; 58: 28-33.

19. Hypertension Study Group. Prevalence, awareness, treatment and control of hypertension among the elderly in Bangladesh and India: a multicentre study. Bull World Health Organ. 2001;79(6):490-500.
Cite this article as: Mani Prasad Gautam, Usha Ghimire,Krishna Maya Shrestha, et al. Prevalence, awareness and control of hypertension in a well-educated professional group in Nepal. Nepalese Heart Journal 2017; 14(1): 21-24 